\title{
Residual Stress-induced Distortions in Laser Powder Bed Additive Manufacturing of Nickel-based Superalloys
}

\author{
Özgür Poyraz, ,* $^{-}$Melih Cemal Kuşhan² \\ 1TEI, Tusaş Engine Industries Inc., Turkey \\ 2Eskişehir Osmangazi University, Faculty of Engineering, Department of Mechanical Engineering, Turkey
}

One of the significant process limits of laser powder bed additive manufacturing technologies is residual stresses and distortions induced by heating the fine metal powder to the melting point and sudden cooling to the initial temperature. While this phenomenon applies to most types of metal alloys, it becomes more important in nickel-based superalloys, which have low thermal conductivity and high-quality requirements for aerospace utilization. Together with the mechanical integrity and geometric problems in the final product, residual stress-induced distortions carry the potential risk of interrupting the job process above a certain distortion limit as a result of part-to-re-coater rubbing. Process simulations performed in a computer environment are of critical importance to predict and to prevent this risk. However, simulation models need to be verified through experiments, and they need to be cost-effective in terms of simulation times. This paper presents a comprehensive study to introduce a fast thermo-mechanical simulation model, together with experiments on nickel-based superalloy demonstrators. It also contributes to the scientific knowledge base with novel coupon design, manufacturing and evaluation methodology for distortion studies.

Keywords: additive manufacturing, finite element analysis, laser powder bed fusion, thermo-mechanical simulations

Highlights

- A fast thermo-mechanical finite element model was introduced for the analysis of distortions and residual stresses in laser powder bed additive manufacturing.

- Novel benchmark coupons were designed for the benchmarking of residual stress-induced distortions considering circumferential effects.

- $\quad$ The thermo-mechanical finite element model was employed for the simulation of benchmark coupons.

- Experiments were conducted for an Inconel 718 nickel-based superalloy using a laser powder bed fusion additive manufacturing system

- Results were evaluated via a coordinate measuring machine equipped with a laser scanner head.

- Successful convergence was achieved with the experimental results.

\section{INTRODUCTION}

Laser powder bed fusion (LPBF) is a powder bed additive manufacturing (AM) process used for the production of three dimensional (3D) parts from a wide range of powder materials; it is known with different commercial names, such as selective laser melting (SLM), selective laser sintering (SLS) or direct metal laser sintering (DMLS) [1]. It expands its sectorial utilization by offering good mechanical properties, design freedom [2], easy transition from design to manufacturing, post-processing ability, and low material consumption [3]. Regardless of the various advantages offered by LPBF, remaining challenges such as surface quality [3], residual stresses, distortions [4], or the use of support structures prevent the wider acceptance of this process.

LPBF AM uses a laser beam to selectively melt the metal powders by scanning cross sections on the surface of a powder bed layer by layer into an object that has a desired 3D shape based on a computer-aided design (CAD) model [5]. After each cross-section is scanned through a mirror system and solidifies with the help of blowing inert gas, the powder bed is lowered by one layer of thickness, a new layer of material is spread on top with a re-coater, and the process is repeated until the part is complete [4]. In this respect, heat transfer mechanisms include phase change, conduction, convection, and radiation (Fig. 1). As a result of the above-mentioned phenomenon, occurring thermal gradients cause cyclic thermal expansions, which exceed the elastic strain of the material and generate plastic strains, especially at high temperatures. These cumulated strains can generate internal stresses (also known as residual stresses) in the part [6] and cause distortions, which may lead failure by cracking [7] or layer delamination [8].

To eliminate the risk of cyclic thermal expansions and the resultant problems of residual stresses and distortions, several measures can be taken. In this regard, to check residual stresses, inspection techniques can be employed such as neutron diffractometry [9] or X-ray diffraction [10], and distortions with dimensional measurement systems [11] can be used. However, due to the rapid nature of additive manufacturing, these techniques are not 
available for in-process control but are commonly used for the final product. Most of the time, detected problems of residual stresses and distortions [12] are overcome on the final product using additional operations, such as stress relieving and/or machining adding extra costs on the product. Moreover, in certain cases, these additional operations may not be useful owing to high stresses or large distortions. On the other side, they are still insufficient to eliminate in-process risks, such as delamination, cracking, or job interruption, due to part-to-re-coater rubbing.

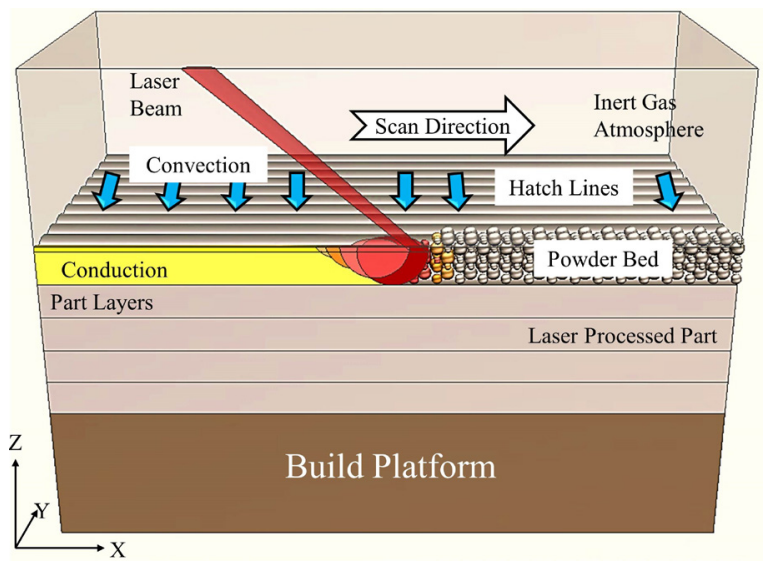

Fig. 1. Heat transfer mechanisms occur as the result of the process [4]

For these reasons, computer-based numerical simulation tools or methods are developed and used to predict residual stresses and distortions. Within the literature, studies on LPBF AM simulations mainly focus on numerical modelling to understand the process itself and the effect of process parameters on the quality of the produced parts.

The state-of-the-art in this field includes different studies and applications on various problems. Accordingly, thermal models coupled with mechanical ones can be used to numerically simulate LPBF processes to understand the effect of different process parameters [13]. The results of these simulations may include the shape and size of the melt pool, temperature distribution, as well as residual stresses and deformations [14]. While this approach is useful for meso-scale process simulations, it may be computationally expensive for macroscale ones [15]. To overcome this computational expense, several researchers have employed diverse approaches. In the beginning phases of additive manufacturing simulations, researchers focused on reducing the mesh count and thus the computation time by using the element birth-and-death technique provided by the general-purpose finite element method (FEM) software packages such as ABAQUS [16] and ANSYS [17]. However, due to the lack of a recordable time reduction in the simulation times, there was a tendency towards other methods in the following periods. The applied plastic strain method is one of the most commonly used methods, using highresolution models of the transient thermo-mechanical analysis, it calculates plastic strain tensor components with equivalent plastic strain and maps the obtained plastic strains on the complete part [18].

This study deals with this challenge by introducing a fast thermo-mechanical finite element model. Together with the proposed model, experimental studies were also conducted on complex parts made of nickel-based superalloys. In this regard, innovative circumferential coupon designs were employed to promote a comprehensive understanding of residual stress induced distortion phenomenon. Verification of the simulation model was accomplished using experimental values.

The rest of the paper is organized as follows: In the next section, details of the thermo-mechanical model were presented among with the coupon geometry designs, necessary material properties and boundary conditions for finite element analysis. The following section explains the experimental procedure in terms of manufacturing and inspection steps. Findings are discussed in the subsequent section with the comparison of modelling results versus experiments. The last section summarizes the paper and emphasizes the potential work to be done.

\section{METHODS}

\subsection{Coupon Designs}

Various types of coupon designs can be utilized to understand the residual stress-induced distortions as a result of LPBF AM. Due to the longer computation times, previous studies used simplistic coupon designs of prismatic shapes to have a constant cross-section along the part [19]. In a few studies, which employs complex geometry, it can be seen that unidirectional cantilever beams are used with arrayed combs to allow distortions [20]. Although these provide useful information about the distortions as a result of LPBF $\mathrm{AM}$ and process parameters, they are not sufficient to reflect different aspects such as the multi-directional differences of distortions due to re-coater movement and/or inert gas blowing. Multi-directional situations were also considered in other previous research, 
but they still lack the evaluation of asymmetry as a significant issue [21].

In the context of this research, innovative coupon geometries were designed with two configurations. These are circumferentially arrayed cantilever beams with $120^{\circ}$ between each beam. The first configuration (C1) employs combs on all beams for investigating the symmetrical situation in terms of distortion results. The second configuration (C2) employs combs on two beams and one of the beams as a full solid without any comb. This is to see the effect of asymmetry on the geometry and also unbalanced material volumes on the parts to effect residual stress-induced distortions. The external dimensions of both coupons were kept equivalent for a fair benchmark, and these dimensions were also kept as low as possible for minimum material consumption. Each coupon has an outer diameter of $70 \mathrm{~mm}$ and each beam has outer dimensions of $35 \mathrm{~mm} \times 10 \mathrm{~mm} \times 7 \mathrm{~mm}$. Combs have a thickness of $1 \mathrm{~mm}$ and a height of $4.5 \mathrm{~mm}$. A $1 \mathrm{~mm}$ gap is present between each comb. Fig. 2 shows the coupons with external dimensions.

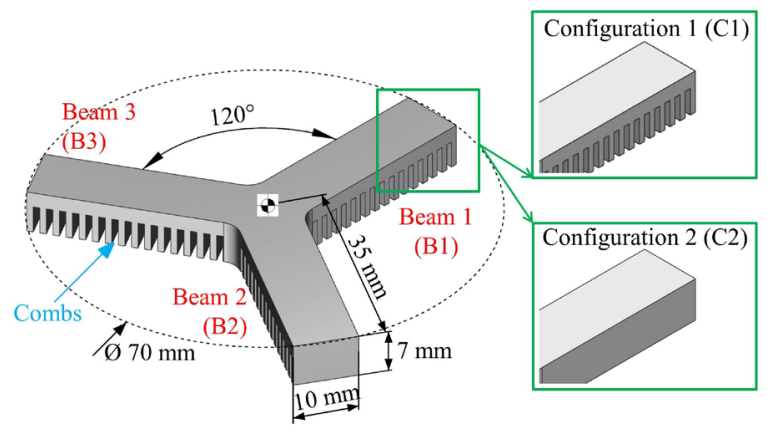

Fig. 2. Circumferential coupons and dimensions

\subsection{Materials and Properties}

Among various LPBF processed superalloys, Inconel 718 was chosen for the material; it has major elements of nickel (52.5\%), chromium (19\%), iron (balance), and niobium $(5.12 \%)$. It possesses both oxidation resistance and high strength at elevated temperatures. Moreover, different forms of Inconel 718 are easily producible with conventional and non-conventional processes, such as machining, welding, and additive manufacturing [22]. Inconel 718, which is preferred in different sectors with its many advantages in terms of design and production, is used in the highest ratio for aircraft engines. An example of this is the PW4000 engine from Pratt \& Whitney [22]. The total ratio of nickel alloys used in the example engine, which provides power to the commercial platforms such as
Boeing 747, Boeing 767, and Airbus 300, is $39 \%$, and the total utilization rate of Inconel 718 in these nickel alloys is $57 \%$.

In this study, EOS Nickel Alloy IN718 was considered for the part material, and all the necessary material properties (physical, thermal, mechanical) for modelling were calculated with $\mathrm{JMatPro}^{\circledR}$ software. According to the proposed methodology, which is explained in the following section, the fast thermomechanical model predicts the distortions in the solid phase and uses material properties below melting temperatures.

The physical phenomena of the process were taken into account, and elasto-plastic behaviour was assumed for the material. Within the elastic region, linear elasticity calculations were conducted using the elastic modulus and Poisson ratio. For the plastic region, von Mises yield criteria were followed, and the temperature dependence of yield strength was adopted. Moreover, the bilinear hardening rule was included using the tangent modulus. Essential properties are given in Table 1 for three different temperature levels. The temperatures between this range and below melting point were extrapolated using polynomial curve fitting of second order.

Table 1. Material properties of as-built IN718

\begin{tabular}{lccc}
\hline Material property $\left[{ }^{\circ} \mathrm{C}\right]$ & 20 & 400 & 600 \\
\hline Density $\left[\mathrm{kg} / \mathrm{m}^{3}\right]$ & 8195 & 8100 & 8000 \\
\hline Thermal conductivity $[\mathrm{W} /(\mathrm{m} \cdot \mathrm{K})]$ & 11.25 & 13.45 & 15.55 \\
\hline Specific heat capacity $[\mathrm{J} /(\mathrm{kg} \cdot \mathrm{K})]$ & 415 & 495 & 580 \\
\hline Thermal expansion $\times 10^{-6}[1 / \mathrm{K}]$ & 13.0 & 14.8 & 16.6 \\
\hline Elastic modulus $[\mathrm{GPa}]$ & 172 & 156 & 143 \\
\hline Poisson ratio $[-]$ & 0.29 & 0.28 & 0.27 \\
\hline Yield strength $[\mathrm{MPa}]$ & 707 & 512 & 260 \\
\hline Tangent modulus $[\mathrm{MPa}]$ & 920 & 785 & 603 \\
\hline
\end{tabular}

\subsection{Modelling}

To overcome the challenge of computationally expensive LPBF AM modelling, a fast thermomechanical finite element model was introduced based on previous studies [13] and [15]. The model was developed using COMSOL Multiphysics FEM software as a result of a series of studies. In the scope of initial studies, a meso-scale model based on a moving heat source with Gaussian distribution was adopted just for thermal simulations [13] and for IN718 material was verified through microstructural investigations on the melt pool dimensions [15]. The convergence of less than $10 \%$ was achieved with the meso-scale model (Fig. 3). 


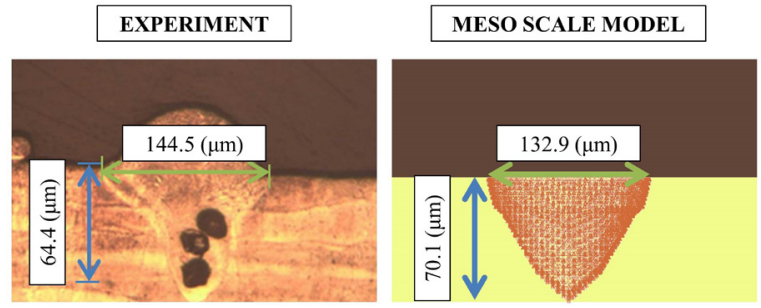

Fig. 3. Results of previously developed meso-scale model [15]

Later, comprehensive parametric analyses were conducted, and the model was accelerated to be used in macro-scale problems having real part geometries [15]. In this regard, the meso-scale model was utilized for various scanning strategies such as band, island, and spiral scanning. Average energy input per unit area and temperature distribution were extracted from the results of these studies. Equivalent energy input was applied to whole layers as a surface heat source simultaneously during the build process by multiplying the energy input per unit area value with the area value of each layer (Fig. 4). The time for the application of the energy input was calculated through dividing surface area value of each layer by the hatch distance multiplied with laser scanning speed (Fig. 4).

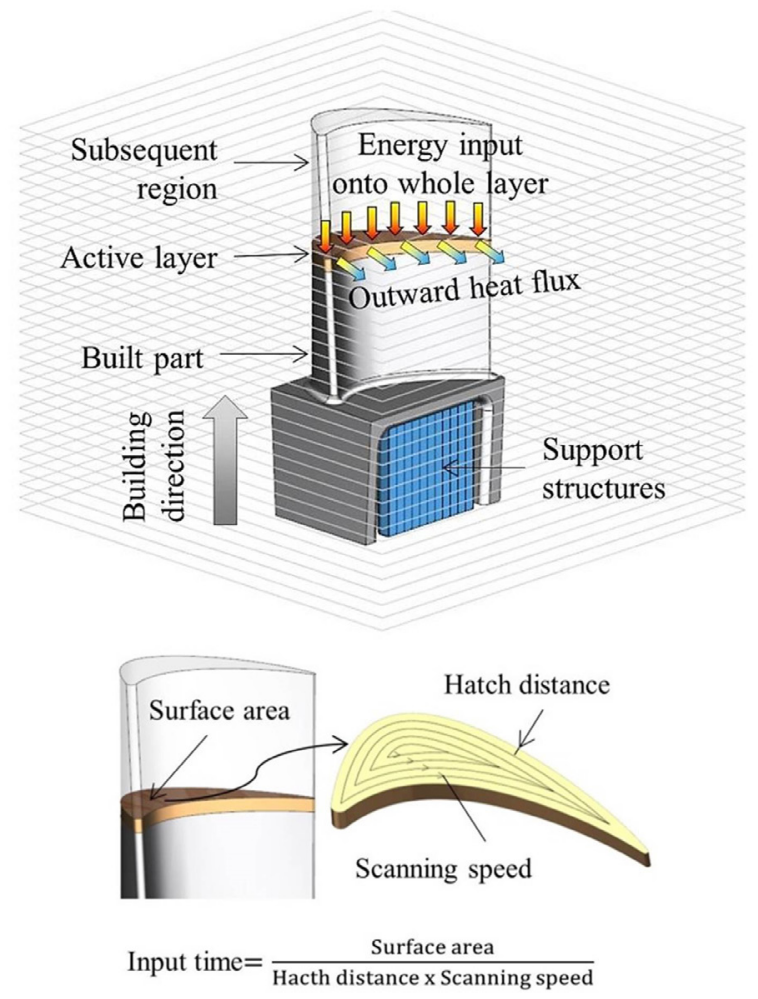

Fig. 4. Fast thermo-mechanical model and input time calculation
Temperature rise due to high loading was reported to be between $5{ }^{\circ} \mathrm{C}$ to $30{ }^{\circ} \mathrm{C}$ in the literature [23]. Since there is low-level loading in AM due to low strain rates and the temperature levels in $\mathrm{AM}$ process are extremely high, such as over $1000{ }^{\circ} \mathrm{C}$ comparing to 5 ${ }^{\circ} \mathrm{C}$ to $30{ }^{\circ} \mathrm{C}$, the effect of deformation on temperature change was neglected. A one-way or weakly coupled thermo-mechanical model was developed, and multiphysics simulations were conducted accordingly. In this type of modelling strategy, the temperature distribution as the result of energy input and heat transfer was mapped simultaneously and transiently to structural mechanics in the same FEM software.

\subsection{Boundary Conditions}

The designed coupon geometries among with the calculated material properties were used for the modelling study, employing the developed fast thermo-mechanical model.

For the thermal boundary conditions, the initial condition at time $t=0$ was set to $20^{\circ} \mathrm{C}$, and the bottom surface temperature of the part geometry was set to 80 ${ }^{\circ} \mathrm{C}$ to simulate the actual condition of build platform pre-heating step. As indicated in the modelling section, equivalent energy input was applied to whole layers as a surface heat source and a convective heat flux condition with a heat transfer co-efficient of $0.0178 \mathrm{~W} /\left(\mathrm{m}^{2} \cdot \mathrm{K}\right)$ [24] was also applied to simulate the effect of argon flow inside the build chamber. The thermal insulation condition was applied for the side surfaces of the part geometry. The reason for this that the side faces of the part geometry are surrounded by powder material, and its thermal conductivity is extremely low [13] and can be neglected comparing it to solid material.

A fixed constraint was assigned to bottom surfaces of the coupon parts. Layer addition was also taken into account with the moving mesh algorithm of the layer plane. For this purpose, the part geometry was modelled at full height, and the block envelope for the geometry was also generated. A predefined Boolean operation (subtract) of block envelope from part geometry was assigned, and the block envelope was transiently transformed with a step function to consider $0.04 \mathrm{~mm}$ layer thickness as a step movement interval and also the layer time as explained in the modelling section. In the final step of simulations, the fixed constraint was deactivated to reflect the real situation of part separation from the build platform.

Mesh size was utilized as the result of mesh sensitivity analyses to allow proper heat dissipation through layer plane and through the side faces. In this 
regard, constant mesh distribution was maintained on the layer plane to facilitate a minimum of two mesh elements for related geometry width and a minimum of five mesh elements for geometry length. Mesh elements on side faces were maintained with a geometric sequence distribution function to remesh the part and update a fine mesh size beneath the layer plane and coarsen the elements close to the part bottom for each layer addition. With this aim, the layer plane surface of the part was meshed with fixed sized triangular elements, and the rest of the geometry was swept with tetrahedral elements. The size of the tetrahedral elements was limited to a minimum value of $0.25 \mathrm{~mm}$ and a maximum value of $5 \mathrm{~mm}$. An element growth ratio of 1.25 was defined to regulate the mesh growth from the minimum to maximum value in each build stage (Fig. 5).

The adaptive solver algorithm feature of the software package was used to transfer nodal history variables for each re-meshing step. For this case of a transient situation, the solution at a certain time interval (of a certain part layer) to the partial differential equation problem on the previous mesh was mapped to the new mesh of the following time interval step. This cycle continued automatically until the end of the simulations.
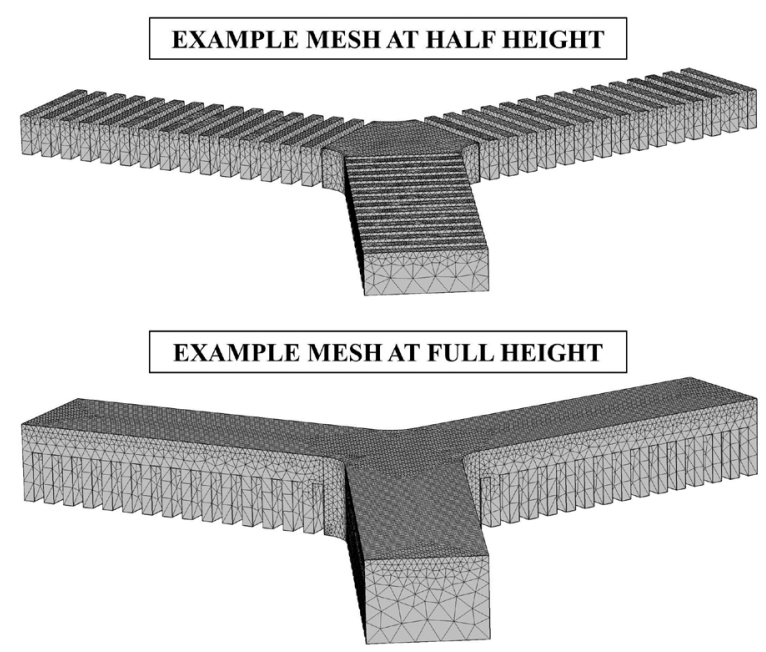

Fig. 5. Mesh examples in different part heights

\subsection{Computations}

The computational studies were conducted in a notebook computer with a central processing unit that had two physical cores and four logical cores with hyper-threading option by a clock speed of 2.40 GHz. Moreover, the computer featured 16 GB of random access memory to facilitate random storing of intermediate simulation data resulting because of the transient iterative solver.

Benchmark studies were conducted by using the above-explained hardware. First, the full layer of the part geometry was simulated using the mesoscale model, and the computation time was 3 hours and 16 minutes. Later, the meso-scale model was used for energy input calibration on a reference unit geometry, ending in 21 minutes. Finally, the simulation for the whole part geometry was conducted in 1 hour 7 minutes. With the proposed fast modelling strategy, the overall computation time was the sum of the calibration, and the whole part simulation and the value was 1 hour 28 minutes. The whole part simulation with the meso-scale model was not conducted, since it could be seen from the one layer simulation that it would take hundreds of hours. From this benchmark, it is obvious that the introduced fast thermo-mechanical model represents considerable benefits in terms of computational expense.

\section{EXPERIMENTAL}

\subsection{Part Manufacturing}

The designed coupons are manufactured in EOS M290 machine of EOS IN718 metal powder. To eliminate any collision or rubbing of coupon parts with the powder re-coater, one of the arms of the coupon was aligned with the $\mathrm{X}$-axis and then rotated $5^{\circ}$.

Laser scanning strategies were adjusted for a proper comparison between the modelling and experimental studies, considering the fact that simulation was conducted with an accelerated thermo-mechanical model that simultaneously applies equivalent energy input to whole layer surface. In this respect, parallel scanning was chosen as the scanning strategy, and the angles of the scanning paths were rotated by a certain degree in each layer so that the

Table 2. Process parameters for IN718

\begin{tabular}{lc}
\hline Parameter & Value \\
\hline Laser power & $285 \mathrm{~W}$ \\
\hline Laser scanning speed & $960 \mathrm{~mm} / \mathrm{s}$ \\
\hline Hatch distance & $0.11 \mathrm{~mm}$ \\
\hline Layer thickness & $0.040 \mathrm{~mm}$ \\
\hline Stripe width & $10.0 \mathrm{~mm}$ \\
\hline Stripe overlap & $0.12 \mathrm{~mm}$ \\
\hline Hatching & $X \& Y$ \\
\hline Strategy & Alternating \\
\hline Angle & $67^{\circ}$ \\
\hline
\end{tabular}


heat distribution did not intensify anywhere through the building process.

Essential LPBF processing parameters are given in Table 2.

\subsection{Part Measurement}

Part measurements were conducted after the parts were removed from the building platform by cutting with an electric discharge machine. The distorted parts as a result of relieving residual stresses after removal from building platform were measured with a Nikon Altera coordinate measuring machine equipped with a non-contact laser scanner (Fig. 6). For a detailed evaluation, many points were obtained during measurement, and the resultant point cloud was exported in stereolithography (STL) file format.

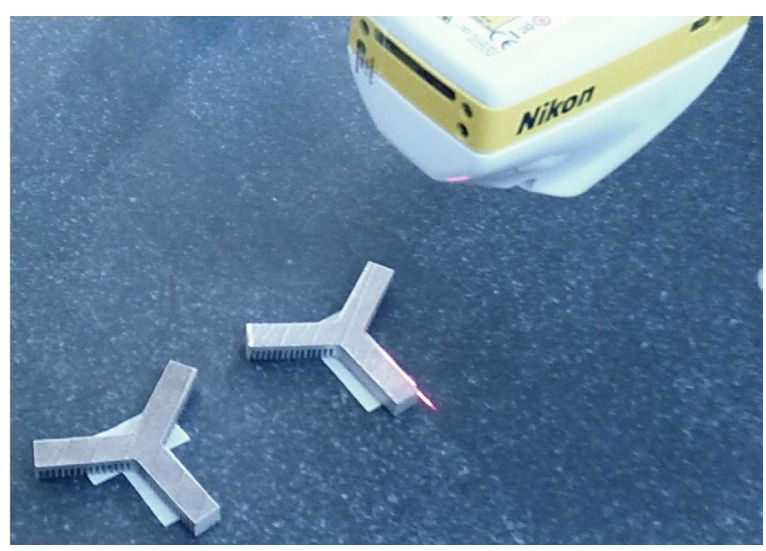

Fig. 6. Part measurement with a non-contact laser scanner

\section{RESULTS AND DISCUSSIONS}

The results of the study were evaluated and discussed from two different perspectives. In the first perspective, modelling and experimental results were compared with each other to understand the convergence of the proposed modelling methodology. In the second perspective, experimental results were discussed for a better understanding of the process and the use of proposed coupon designs for residual stress induced distortion evaluation.

Finite element analyses were performed for the proposed model following to assignment of part geometry, material properties and boundary conditions, and outputs were obtained as part deformation (Fig. 7). Deformed part geometry was also exported in STL file format compatible to measurement results. Both STL files (one from measurement results and one from modelling) were imported to single CAD software for comparison purposes, and both geometries were aligned from their top faces.

The comparison was made using the height ( $\mathrm{Z}$ value) references of edges at the end of each beam. Modelling and measurement results are presented in Table 3. In Table 3, the convergence ratio was calculated as the ratio of the measurement to the modelling difference divided by measurement value.

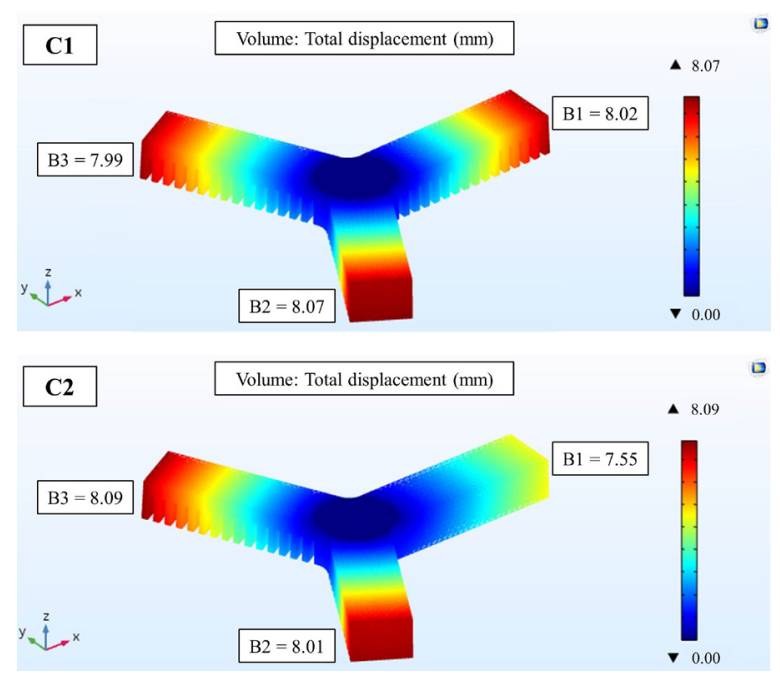

Fig. 7. Example of the simulation results and reference edge numbers of coupons

Table 3. Comparison of modelling and experimental results

\begin{tabular}{lccc}
\hline $\begin{array}{l}\text { Reference } \\
\text { edge }\end{array}$ & $\begin{array}{c}\text { Modelling } \\
{[\mathrm{mm}]}\end{array}$ & $\begin{array}{c}\text { Measurement } \\
{[\mathrm{mm}]}\end{array}$ & $\begin{array}{c}\text { Convergence ratio } \\
{[\%]}\end{array}$ \\
\hline C1-B1 & 8.02 & 8.34 & 3.8 \\
\hline C1-B2 & 8.07 & 8.31 & 2.8 \\
\hline C1-B3 & 7.99 & 8.27 & 3.3 \\
\hline C2-B1 & 7.55 & 8.06 & 6.3 \\
\hline C2-B2 & 8.01 & 8.38 & 4.4 \\
\hline C2-B3 & 8.09 & 8.41 & 3.9 \\
\hline
\end{tabular}

As can be seen from Table 3, the convergence between the modelling and measurement results is better than $10 \%$, based on the overall height of the edges of both coupons. The success of the convergence was obtained as the result of the proposed modelling method, since the modelling method considers equivalent energy input similar to the real situation utilized by parallel scanning with alternating angles. The proposed modelling method can also be used for the processes utilizing island (chessboard) or spiral scanning strategies [3]. Nevertheless, convergence in fixed-angle parallel or zigzag scans is not expected 
to be as successful due to the remarkable thermal gradients between $\mathrm{X}$ and $\mathrm{Y}$ directions [19].

The experiments with the use of proposed coupon designs have also revealed noteworthy results. It can be observed from the results of coupon $\mathrm{C} 1$ that all deformation values in reference edges of B1, B2 and B3 are considerably close to each other, within a few microns. In contrast, it is evident from the results of coupon $\mathrm{C} 2$ that the distortion results of the reference edge B1 of the full beam is noticeably lower than B2 and $\mathrm{B} 3$, with $0.3 \mathrm{~mm}$ between each.

According to the extracted deduction from this study, it can be said that the effect of variables such as gas blow or powder re-coating direction is low in terms of distortion values of the same geometries in different layout positions and/or angles. In contrast, the difference in distortion values of geometries with varying features is high. In light of this information, it is recommended to reinforce the specific geometrical features in cases in which the residual stress-induced distortion problems are experienced and cannot be solved by the different positioning of the part.

\section{CONCLUSIONS}

One of the process limits of LPBF AM is residual stress induced distortions to expose mechanical integrity and geometric problems in the final product. Compared to other metallic materials, this problem becomes more important for nickel-based superalloys having low thermal conductivity and high quality requirements for aerospace utilization. The lack of in-process measurement techniques, difficulty in overcoming resultant problems and the risk of job failures show the importance of employing computerbased numerical simulation tools or methods to predict residual stresses and distortions.

This study introduced a fast thermo-mechanical finite element model together with experiments conducted on complex parts made of nickel-based superalloys, in this case, Inconel 718. Unlike previous studies in the literature, innovative distortion coupon designs were also presented to facilitate a comprehensive understanding of circumferential effects during process.

The introduced thermo-mechanical model provided a computationally feasible method and showed good convergence results, better than 10 $\%$ for the case of strategy to rotate scanning vectors with an alternating angle. Via the equivalent energy input principle, the proposed method can also be used for the processes utilizing island or spiral scanning strategies.
The experimental results revealed that geometrical difference are mostly dominant in comparison to circumferential effects such as gas blow or powder recoater on residual stresses and residual stress induced distortions.

Future studies may include detailed measurement of residual stresses using x-ray diffraction techniques, research on other nickel-based superalloys, parametric simulations on representative part geometries and consideration of other scanning strategies, such fixedangle parallel or zigzag scans, by improving the modelling method.

\section{ACKNOWLEDGEMENTS}

The authors would like to thank TEI (Tusaş Engine Industries Inc.) for supporting this research.

\section{REFERENCES}

[1] Levy, G.N., Schindel, R., Kruth, J.P. (2003). Rapid manufacturing and rapid tooling with layer manufacturing (LM) technologies, state of the art and future perspective. CIRP Annals-Manufacturing Technology, vol. 52, no. 2, p. 589-609, DOI:10.1016/S0007-8506(07)60206-6.

[2] Thompson, M.K., Moroni, G., Vaneker, T., Fadel, G., Campbell, R.I., Gibson, I., Bernard, A., Schulz, J., Graf, P., Ahıja, B., Martina, F. (2016). Design for additive manufacturing: trends, opportunities, considerations, and constraints. CIRP Annals-Manufacturing Technology, vol. 65, no. 2, p. 737-760, DOI:10.1016/j.cirp.2016.05.004.

[3] Poyraz, Ö., Kuşhan, M.C. (2018). Investigation of the effect of different process parameters for laser additive manufacturing of metals. Journal of the Faculty of Engineering and Architecture of Gazi University, vol. 33, no. 2, p. 729-742, D0I:10.17341/gazimmfd.416479.

[4] Kuşhan, M.C., Poyraz, Ö., Uzunonat Y., Orak, S. (2018). Systematical review on the numerical simulations of laser powder bed additive manufacturing. Sigma Journal of Engineering and Natural Sciences, vol. 36, no. 4, p.11971214.

[5] Guo, N., Leu, M.C. (2013). Additive manufacturing: technology, applications and research needs. Frontiers of Mechanical Engineering, vol. 8, no. 3, p. 215-243, Dol:10.1007/s11465013-0248-8.

[6] Van Belle, L., Vansteenkiste, G., Boyer, J.C. (2012). Comparisons of numerical modelling of the Selective Laser Melting. Key Engineering Materials, vol. 504-506, p. 10671072, D0I:10.4028/www.scientific.net/KEM.504-506.1067.

[7] Mercelis, P., Kruth, J.P. (2006). Residual stresses in selective laser sintering and selective laser melting. Rapid Prototyping Journal, vol. 12, no. 5, p. 254-265, D0l:10.1108/13552540610707013.

[8] Nickel, A., Barnett, D., Link, G., Prinz, F. (1999). Residual stresses in layered manufacturing. Proceedings of the solid Freeform Fabrication Symposium, p. 239-246. 
[9] Zaeh, M.F., Branner, G. (2010). Investigations on residual stresses and deformations in selective laser melting. Production Engineering, vol. 4, no. 1, p. 35-45, D0l:10.1007/ s11740-009-0192-y.

[10] Vogel, M., Khan, M., Medina, J.l., Pinkerton, A., N'Dri, N., Megahed, M. (2012). A verified model of transient and residual stresses in Laser Direct Metal Deposition. International Congress on Applications of Lasers and Electro-Optics, p. 8996, DOI:10.2351/1.5062560.

[11] Yang, Y.P., Jamshidinia, M., Boulware, P., Kelly, S.M. (2017). Prediction of microstructure, residual stress, and deformation in laser powder bed fusion process. Computational Mechanics, vol. 61, no. 5, p. 599-615, D0l:10.1007/s00466-017-1528-7.

[12] Piaget, A., Museau, M., Paris, H. (2017). Manufacturing space homogeneity in additive manufacturing - Electron beam melting case. Strojniški vestnik - Journal of Mechanical Engineering, vol. 63, no. 10, p. 553-558, D0l:10.5545/svjme.2017.4365.

[13] Kundakcıoğlu, E., Lazoglu, I., Poyraz, Ö., Yasa, E., Cizicioğlu, N. (2018). Thermal and molten pool model in selective laser melting process of Inconel 625. The International Journal of Advanced Manufacturing Technology, vol. 95, no. 9-12, p. 3977-3984, DOI:10.1007/s00170-017-1489-1.

[14] Andreotta, R., Ladani, L., Brindley, W. (2017). Finite element simulation of laser additive melting and solidification of Inconel 718 with experimentally tested thermal properties. Finite Elements in Analysis and Design, vol. 135, p. 36-43, D0l:10.1016/j.finel.2017.07.002.

[15] Poyraz, Ö. (2018) Investigation of the Effects of Process Parameters for Laser Additive Manufacturing by Using Modeling and Simulation Methods, PhD Thesis, Eskişehir Osmangazi University, Eskişehir.

[16] Ding, J., Colegrove, P., Mehnen, J., Ganguly, S., Sequeira Almeida, P.M., Wang, F., Williams, S. (2011). Thermomechanical analysis of Wire and Arc additive layer manufacturing process on large multi-layer parts.
Computational Materials Science, vol. 50, no. 12, p. 33153322, D0I:10.1016/j.commatsci.2011.06.023.

[17] Zekovic, S., Dwivedi, R., Kovacevic, R. (2005). Thermostructural finite element analysis of direct laser metal deposited thin-walled structures. Proceedings SFF Symposium, Austin.

[18] Keller, N., Ploshikhin, V. (2014). New method for fast predictions of residual stress and distortion of AM parts. Solid Freeform Fabrication Symposium, Austin p. 4-6.

[19] Shukla, M., Verma, V. (2014). Finite element simulation and analysis of laser metal deposition, 6 ${ }^{\text {th }}$ International Conference on Mechanical, Production and Automobile Engineering, Cape Town.

[20] Neugebauer, F., Keller, N., Xu, H., Kober C., Ploshikhin, V. (2014). Simulation of selective laser melting using process specific layer based meshing. Proceedings of Fraunhofer Direct Digital Manufacturing Conference, Aachen.

[21] Sillars, S.A., Sutcliffe, C.J., Philo, A.M., Brown, S.G.R., Sienz, J., Lavery, N.P. (2018). The three-prong method: a novel assessment of residual stress in laser powder bed fusion. Virtual and Physical Prototyping, vol. 13, no. 1, p. 20-25, DOl:10.1080/17452759.2017.1392682.

[22] Paulonis, D.F., Schirra, J.J. (2001). Alloy 718 at Pratt \& WhitneyHistorıcal Perspectıve and Future Challenges. TMS Superalloys 718. 625. 706 and Various Derivatives, D0I:10.7449/2001/ Superalloys_2001_13_23.

[23] Tao, J., Zhang, H., Zheng, Y., Chen, Z. (2018). Development of generalized interpolation material point method for simulating fully coupled thermomechanical failure evolution. Computer Methods in Applied Mechanics and Engineering, vol. 332, p. 325-342, DOl:10.1016/j.cma.2017.12.014.

[24] Lemmon, E.W., Jacobsen, R.T. (2004). Viscosity and thermal conductivity equations for nitrogen, oxygen, argon, and air. International Journal of Thermophysics, vol. 25, no. 1, p. 2169, DOI:10.1023/B:IJOT.0000022327.04529.f3. 